\title{
Instituições que recebem a revista Horizonte como Doação
}

1 Bibliografia Bíblica Latino-Americana

2 Biblioteca do Palácio do Governo de Santa Catarina

3 Centro de Ensino Superior de Juiz de Fora - CESJF

4 Centro de Ensino Superior de São Carlos

5 Centro de Ensino Superior do Brasil - CESB

6 Centro de Formação Religiosa Superior do Estado do Maranhão - CEFRES

7 Centro Universitário Católico Salesiano Auxilium U.E. - UNISALESIANO

8 Centro Universitário Claretiano - CEUCLAR

9 Centro Universitário da FEI

10 Centro Universitário de João Pessoa - UNIPÊ

11 Centro Universitário Diocesano do Sudoeste do Paraná - UNICS

12 Centro Universitário do Leste de Minas - UNILESTE

13 Centro Universitário Franciscano do Paraná

14 Centro Universitário Luterano de Palmas

15 Centro Universitário Salesiano Auxilium SEDE - UNISALESIANO

16 Centro Universitário Salesiano Auxilium U.E. Lins - UNISALESIANO

17 Centro Universitário Salesiano de São Paulo - SEDE

18 Centro Universitário Salesiano de São Paulo - U.E. Lorena

19 Centro Universitário Salesiano de São Paulo - UNISAL

20 Centro Universitário São Camilo

21 Escola de Enfermagem Wenceslau Braz - EEWB

22 Faculdade Batista Brasileira - FBB

23 Faculdade Católica de Uberlândia

24 Faculdade Damas

25 Faculdade de Ciência e Educação de Rubiataba - FACER 
26 Faculdade de Ciências da Bahia - FACIBA

27 Faculdade de Filosofia Santa Dorotéia - FFSD

28 Faculdade de Filosofia São Miguel Arcanjo - FAFISMA

29 Faculdade de Filosofia, Ciências e Letras Santa Marcelina

30 Faculdade Evangélica do Meio Norte - FAEME

31 Faculdade Evangélica do Paraná

32 Faculdade Frassinetti do Recife - FAFIRE

33 Faculdade João Paulo II - FAJOPA

34 Faculdade La Salle - UNILASALLE

35 Faculdade Salesiana do Nordeste - FASNE

36 Faculdade Salesiana Dom Bosco

37 Faculdade Santa Marcelina

38 Faculdade Santíssimo Sacramento - FSSS

39 Faculdade São Bento da Bahia

40 Faculdade São Luiz

41 Faculdades Integradas Coração de Jesus

42 Fundação Biblioteca Nacional

43 Fundação Universidade Estadual de Maringá - FUEM

44 Instituto de Desenvolvimento Cultural - IDC

45 Instituto Filosófico Teológico Nossa Senhora Imaculada

46 Instituto Presbiteriano Mackenzie

47 Instituto Santa Tereza

48 Instituto Santo Tomás de Aquino - ISTA

49 Instituto Teológico Arquidiocesano Santo Antônio - ITASA

50 Instituto Teológico Pastoral do Ceará - ITEP

51 Katholieke Universiteit Leuven

52 Pontifícia Universidade Católica de Chile

53 Pontifícia Universidade Católica de Minas Gerais - PUC Minas Guanhães

54 Pontifícia Universidade Católica de Minas Gerais - PUC Minas Poços de Caldas

55 Pontifícia Universidade Católica de Minas Gerais - PUC Minas / Praça da Liberdade

56 Pontifícia Universidade Católica de Minas Gerais - PUC Minas / São Gabriel

57 Pontifícia Universidade Católica de Minas Gerais - PUC Minas / Arcos

58 Pontifícia Universidade Católica de Minas Gerais - PUC Minas / Betim 
59 Pontifícia Universidade Católica de Minas Gerais - PUC Minas / Contagem

60 Pontifícia Universidade Católica do Rio de Janeiro - PUC-RJ

61 The U.S.Library of Congress Office, Brazil

62 Universidad Complutense de Madrid

63 Universidad Nacional Autônoma de México - UNAM

64 Universidade Católica de Brasília - UCB

65 Universidade Católica de Petrópolis - UCP

66 Universidade Católica do Salvador - UCSAL

67 Universidade Católica Dom Bosco - UCDB

68 Universidade Católica Portuguesa - UCP

69 Universidade Católica Rainha da Paz

70 Universidade de Brasília - UnB

71 Universidade de São Paulo - USP - FFLCH

72 Universidade de Sorocaba - UNISO

73 Universidade do Estado de Minas Gerais - UEMG

74 Universidade do Estado do Rio de Janeiro - UERJ

75 Universidade do Estado do Rio Grande do Norte - UERN

76 Universidade do Sagrado Coração

77 Universidade do Vale do Rio dos Sinos - UNISINOS

78 Universidade do Vale do Sapucaí - UNIVAS

79 Universidade Estadual de Campinas - UNICAMP

80 Universidade Estadual de Feira de Santana

81 Universidade Estadual de Montes Claros - UNIMONTES

82 Universidade Estadual de Santa Cruz

83 Universidade Estadual do Ceará - UECE

84 Universidade Estadual Vale do Acaraú - UVA-CE

85 Universidade Federal da Bahia - UFBA

86 Universidade Federal da Grande Dourados - UFGD

87 Universidade Federal da Paraíba - UFPB

88 Universidade Federal de Alagoas - UFAL

89 Universidade Federal de Mato Grosso - UFMT

90 Universidade Federal do Mato Grosso do Sul - UFMS

91 Universidade Federal de Ouro Preto - UFOP 
92 Universidade Federal de Pernambuco - UFPE

93 Universidade Federal de Santa Catarina - UFSC

94 Universidade Federal de Santa Maria

95 Universidade Federal de São João Del Rei - UFSJ

96 Universidade Federal de Sergipe - UFSE

97 Universidade Federal do Ceará - UFC

98 Universidade Federal do Espírito Santo - UFES

99 Universidade Federal do Maranhão - UFMA

100 Universidade Federal do Pará - UFPA

101 Universidade Federal do Paraná - UFPR

102 Universidade Federal do Piauí - UFPI

103 Universidade Federal do Rio de Janeiro - UFRJ

104 Universidade Federal do Rio Grande do Norte

105 Universidade Gama Filho - UGF

106 Universidade Santa Úrsula - USU

107 Universidade São Francisco - USF

108 Universidade Tecnológica Federal do Paraná - UTFPR

109 Universidade Vale do Rio Doce - UNIVALE

110 Universitat Passau (Alemanha) 